\title{
Molecular Imaging Reveals a High Degree of Cross-Seeding of Spontaneous Metastases in a Novel Mouse Model of Synchronous Bilateral Breast Cancer
}

\author{
Shirley Liu, ${ }^{1,2}$ Nivin N Nyström, ${ }^{1,2}$ John J Kelly, ${ }^{1}$ Amanda M Hamilton, ${ }^{1}$ Yanghao Fu, ${ }^{1,2}$ \\ John A Ronald $\oplus^{1,2}$ \\ ${ }^{1}$ Robarts Research Institute, University of Western Ontario, London, ON, Canada \\ ${ }^{2}$ Department of Medical Biophysics, University of Western Ontario, London, ON, Canada
}

\begin{abstract}
Purpose: Synchronous bilateral breast cancer (SBBC) patients present with cancer in both breasts at the time of diagnosis or within a short time interval. They show higher rates of metastasis and lower overall survival compared to women with unilateral breast cancer. Here we established the first preclinical SBBC model and used molecular imaging to visualize the patterns of metastasis from each primary tumor.

Procedures: We engineered human breast cancer cells to express either Akaluc or Antares2 for bioluminescence imaging (BLI) and tdTomato or zsGreen for ex vivo fluorescence microscopy. Both cell populations were implanted into contralateral mammary fat pads of mice $(n=10)$, and dual-BLI was performed weekly for up to day $29(n=3), 38(n=4)$, or $42(n=3)$. Primary tumors and lungs were fixed, and ex vivo fluorescence microscopy was used to analyze the cellular makeup of micrometastases.

Results: Signal from both Antares2 and Akaluc was first detected in the lungs on day 28 and was present in 9 of 10 mice at endpoint. Ex vivo fluorescence microscopy of the lungs revealed that for mice sacrificed on day 38, a significant percentage of micrometastases were composed of cancer cells from both primary tumors (mean 37\%; range 27 to $45 \%$ ), while two mice sacrificed on day 42 showed percentages of $51 \%$ and $70 \%$.

Conclusions: A high degree of metastatic cross-seeding of cancer cells derived from bilateral tumors may contribute to faster metastatic growth and intratumoral heterogeneity. We posit that our work will help understand treatment resistance and optimal planning of SBBC treatment.
\end{abstract}

Key words: Synchronous bilateral breast cancer, Mouse model, Spontaneous metastases, Metastatic cross-seeding, Dual bioluminescence imaging

\section{Introduction}

Synchronous bilateral breast cancer (SBBC) is defined as the detection of breast cancer in both breasts at the time of

Correspondence to: John Ronald; e-mail: jronald@robarts.ca diagnosis or within a short interval of 3-12 months [1]. SBBC accounts for $2-11 \%$ of all breast cancer cases, and the estimated risk of SBBC development is 2-6 times higher in patients already diagnosed with breast cancer [2,3]. Several clinical studies have found that compared to patients with unilateral breast cancer (UBC), SBBC patients have higher rates of distant metastasis and lower overall survival [2-7]. 
In 2015, Jobsen and colleagues reported that the aggressiveness of primary tumors in SBBC patients does not appear to differ from UBC patients, as measured by malignancy grading, mitotic activity index, hormone receptor status, and the presence of positive lymph nodes [2]. They suggested that the worse prognosis of SBBC is due to the combined effect of two tumors, resulting in a higher chance of metastasis. Using a competing risks model, Mejdahl et al found that the combined effect of having two cancers leads to excess mortality and poorer prognosis in comparison with UBC [7]. These studies suggest that increased tumor burden and metastasis may explain the lower survival of SBBC patients; however, little is known about the spread and growth of metastatic cells from each primary tumor over time.

Animal models of UBC have existed for decades and have provided vital information on the steps, kinetics, and mechanisms of breast cancer metastasis, as well as the development and testing of anti-metastatic treatments [812]. Similar animal models of SBBC are lacking, and to our knowledge, there are no cell lines derived from SBBC patients. Since it is likely that SBBC patients experience worse outcome due to increased tumor burden rather than tumor aggressiveness, we believe SBBC may be modeled with traditional breast cancer cell lines. Thus, to complement the data from clinical SBBC studies, we developed a xenograft mouse model of SBBC where each mouse formed two contralateral primary breast cancer tumors with spontaneous metastasis. Cancer cells implanted into each mammary fat pad were pre-engineered to express a unique set of imaging reporter genes allowing us to distinctly visualize the metastatic fate of cancer cells from each tumor using noninvasive in vivo dual-bioluminescence imaging (BLI) and ex vivo fluorescence microscopy.

BLI relies on the reaction between luciferases and exogenously delivered substrates to produce light that is then detected by a charge-coupled device [13]. In this study, we used two highly sensitive BLI reporters Antares2 and Akaluc to improve our ability to monitor SBBC metastasis in deep tissues. Antares 2 is an enhanced version of the original Antares reporter developed by Dr. Michael Lin's group. Antares is a bioluminescence resonance energy transfer (BRET) reporter protein consisting of the BLI reporter NanoLuc fused to two copies of the orange fluorescent protein CyOFP1 [14]. Reacting with furimazine, a synthetic analog of the Renilla luciferase substrate coelenterazine, NanoLuc emits blue light that can be absorbed by CyOFP1 and re-emitted as orange light (583 nm emission peak), allowing better tissue penetration. Antares 2 was developed by replacing NanoLuc with a mutant named teLuc [15]. Akaluc is a recently described luciferase that also promotes red-shifted light emission (677 nm emission peak) [16]. This BLI reporter was developed by Dr. Atsushi Miyawaki's group through successive rounds of mutagenesis of firefly luciferase to optimize pairing with the substrate AkaLumine hydrochloride [16]. Akaluc has been shown to be able to achieve detection of single cells at depth (lungs) in mice [16]. In addition to these BLI reporters, the fluorescence reporters zsGreen and tdTomato were co-expressed by either Antares 2 or Akaluc-expressing cells, respectively, to enable ex vivo microscopic cell characterization of SBBC metastases [17].

Using this first SBBC model, we aimed to identity the patterns of SBBC metastasis over time. We demonstrate that the vast majority of individual lung metastases are formed from cancer cells derived from both primary tumors rather than from one individual tumor, highlighting a high degree of cross-seeding. If present in SBBC patients, this high level of cross-seeding from distinct primary tumors may contribute to tumor heterogeneity and treatment resistance. Future work using our model and potential variants may help further elucidate the biological mechanisms underlying the worse prognosis of SBBC patients.

\section{Methods}

\section{Lentiviral Construction and Production}

Third-generation lentiviral packaging and envelopeexpression plasmids pMDLg/pRRE, pRSV-Rev, and pMD2.G were gifts from Didier Trono (Addgene plasmids \#12251, \#12253, and \#12259, respectively) [18]. pUltraChili-Luc, a third-generation lentiviral transfer vector encoding tdTomato (tdT) and firefly luciferase (FLuc) separated by a P2A self-cleaving peptide sequence, was a gift from Malcolm Moore (Addgene plasmid \#48688). All cloning was performed using In-Fusion HD Cloning (Takara Bio USA, Inc.). As previously reported [19], we built a pEF1 $\alpha-$ tdT/FLuc2 transfer vector by replacing FLuc with FLuc2 and the ubiquitin $\mathrm{C}$ promoter with a human elongation factor 1 alpha promoter (pEF1 $\alpha$ ) in the pUltra-Chili-Luc vector. To generate a pEF1 $\alpha$-tdT/Akaluc transfer plasmid, the Fluc2 sequence in $\mathrm{pEF} 1 \alpha$-tdT/FLuc2 was replaced with Akaluc obtained from the pcDNA3-Venus-Akaluc Vector (Cat. RDB15781, RIKEN BioResource Research Center). To generate a $\mathrm{pEF} 1 \alpha$-zsG/Antares2 transfer plasmid, the pEF1 $\alpha$-tdT/FLuc2 vector was modified to replace tdT with the fluorescence reporter zsGreen 1 (zsG) obtained from the pLVX-ZsGreen1-N1 Vector (Cat. 632565, Takara Bio USA, Inc.) and FLuc2 was replaced with the Antares2 sequence obtained from pcDNA3-Antares2 c-myc, a gift from Huiwang Ai (Addgene plasmid \#100027) [15]. To produce $\mathrm{pEF} 1 \alpha-\mathrm{zsG} / \mathrm{Antares} 2$ and $\mathrm{pEF} 1 \alpha$-tdT/Akaluc lentiviruses, the packaging, envelope, and one of the transfer plasmids were co-transfected into human embryonic kidney (HEK 293T) cells using Lipofectamine 3000 (Thermo Fisher Scientific) according to the manufacturer's lentiviral production protocol. Lentiviruscontaining supernatants were harvested $24-$ and 48$\mathrm{h}$ post-transfection, filtered through a $0.45-\mu \mathrm{m}$ filter, and stored at $-80^{\circ} \mathrm{C}$ prior to use. 


\section{Cell Culture and Lentiviral Transduction}

Human triple negative breast cancer cells (MDA-MB-231) were obtained from a commercial supplier (American Type Culture Collection; ATCC) and cultured in DMEM supplemented with $10 \%$ fetal bovine serum at $37^{\circ} \mathrm{C}$ and $5 \% \mathrm{CO}_{2}$. All cells were routinely verified as free of mycoplasma contamination using the MycoAlert mycoplasma detection kit (Lonza). Cells were transduced with pEF1 $\alpha$-zsG/Antares2 lentivirus overnight in the presence of $4-$ to $8-\mu \mathrm{g} / \mathrm{ml}$ polybrene. Transduced cells were washed, collected, and sorted for zsG expression using a FACSAria III fluorescence-activated cell sorter (BD Biosciences), generating Antares2-expressing cells with $>95 \%$ purity. A second population of MDA-MB-231 cells were transduced with the pEF1 $\alpha$-tdT/Akaluc lentivirus and sorted for tdT expression to generate Akaluc-expressing cells with $>95 \%$ purity.

\section{Furimazine and AkaLumine- $\mathrm{HCl}$}

Furimazine was purchased as NanoLuc substrate in the Nano-Glo ${ }^{\circledR}$ Luciferase Assay System (Promega) and was diluted $50 \times$ in media for in vitro experiments and $50 \times$ in PBS for in vivo experiments, as previously described [20]. AkaLumine-HCl (TokeOni; Sigma-Aldrich) was diluted in PBS to $5 \mathrm{mM}$ for in vivo experiments. Further dilutions to $250 \mu \mathrm{M}$ were made in media for in vitro experiments.

\section{In Vitro Studies}

Fluorescence microscopy was performed on an Olympus IX50 Inverted System Microscope to visualize zsG in Antares2expressing cells and tdT in Akaluc-expressing cells. To assess Antares2 and Akaluc activity and the correlation between cell number and BLI signal, increasing cell numbers from $1 \times 10^{4}$ to $1.5 \times 10^{5}$ cells were seeded in a 24 -well plate. The next day, media was removed and replaced with $200 \mu \mathrm{l}$ of furimazine diluted $50 \times$ in media for Antares2-expressing cells, or $200 \mu \mathrm{l}$ of $250 \mu \mathrm{M}$ AkaLumine- $\mathrm{HCl}$ in media, and images were acquired 5 min later.

Proliferation assays were performed to evaluate whether genetic engineering had an effect on in vitro cell growth. Naïve cells, Antares2-expressing, and Akaluc-expressing cells were plated in 24 -well plates at $2 \times 10^{4}$ cells per well in triplicate. Viable cell numbers were counted by Trypan Blue dye exclusion at 24, 48, 72, and $96 \mathrm{~h}$.

To evaluate the substrate in vitro cross-reactivity of furimazine with Akaluc and AkaLumine-HCl with Antares2, two 24-well plates were plated with $5 \times 10^{4}$ Akalucexpressing cells, Antares 2 -expressing cells, naïve cells, and equivalent volume of media. The next day, media was removed and replaced with $200 \mu \mathrm{l}$ of $250 \mu \mathrm{M}$ AkaLumine$\mathrm{HCl}$ in media in one plate, and $200 \mu \mathrm{l}$ of furimazine diluted $50 \times$ in media in another plate. Images were acquired $5 \mathrm{~min}$ after incubation. After initial images were acquired, cells were washed with PBS, incubated with $1 \mathrm{ml}$ of fresh media, and signal decay was measured for up to $3 \mathrm{~h}$. Cells were washed again every $24 \mathrm{~h}$ followed by BLI until signal reached background levels. Results were obtained from three independent experiments with three replicates for each condition. The mean signal across replicates was determined for each independent experiment.

\section{In Vivo Studies}

Animals were cared for in accordance with the standards of the Canadian Council on Animal Care and under an approved protocol of the University of Western Ontario's Council on Animal Care (2017-032). Six to eight-week-old female nu/nu mice were obtained from Charles River Laboratories (Willington, MA, USA).

We first evaluated the kinetics of Antares2 and Akaluc in vivo in nu/nu mice. Mice received orthotopic injections of either Antares2-expressing cells $\left(5 \times 10^{5} ; n=4\right)$ or Akaluc-expressing cells $\left(5 \times 10^{5} ; n=4\right)$ into the right fourth mammary fat pad (day 0$)$. On day 5, all mice received an intravenous injection of $100 \mu \mathrm{l}$ of furimazine (diluted 50× in PBS), and images were acquired every $60 \mathrm{~s}$ for $30 \mathrm{~min}$, as well as at $1,2,3$, and $24 \mathrm{~h}$. On day 6 , all mice received intraperitoneal injections of $100 \mu \mathrm{l}$ of $5 \mathrm{mM}$ AkaLumine$\mathrm{HCl}$ in PBS, and images were acquired every $60 \mathrm{~s}$ for $30 \mathrm{~min}$ and then at 1, 2, 7, 8, 11, 24, and $36 \mathrm{~h}$. BLI was performed on an IVIS Lumina XRMS In Vivo Imaging System (PerkinElmer). For all imaging, mice were anesthetized with $1-2 \%$ isoflurane using a nose cone attached to an activated carbon charcoal filter for passive scavenging. Regions of interest (ROIs) were manually drawn around tumor borders using Living Image software to measure bioluminescent average radiance $\left(\mathrm{p} / \mathrm{s} / \mathrm{cm}^{2} / \mathrm{sr}\right)$.

To generate a SBBC model, 8-week-old female NOD scid-gamma (NSG) mice were obtained from an in-house colony (Dr. David Hess; Western University). Each mouse received orthotopic injections of $3 \times 10^{5}$ Antares2-expressing cells into the right fourth mammary fat pad and $3 \times 10^{5}$ Akaluc-expressing cells into the left fourth mammary fat pad (day $0 ; n=10$ ). Antares2 BLI was performed weekly starting day 0 for up to 6 weeks. Images were acquired for up to 10 min. Akaluc BLI was performed on the same or on consecutive days after scans taken prior to AkaLumine$\mathrm{HCl}$ injection confirmed lack of Antares 2 signal. Images were acquired for up to $30 \mathrm{~min}$. Mice 1-3 were sacrificed on day 29 upon detection of both Antares2- and Akalucexpressing lung metastasis, and tissues were collected for histological analysis. The remaining seven mice were monitored for primary tumor growth and metastasis until endpoint, determined by the development of primary tumor necrosis (day 42 for mice 4-6 and day 38 for mice 7-10).

\section{Histology}

At endpoint, mice were sacrificed by isoflurane overdose and perfused with $4 \%$ paraformaldehyde (PFA) via the left ventricle. Mammary fat pad tumors and lungs were removed 
and fixed in 4\% PFA for an additional $48 \mathrm{~h}$, cryopreserved in ascending concentrations of sucrose ( 15 and $30 \% \mathrm{w} / \mathrm{v}$ ) for $24 \mathrm{~h}$ each. Tissues were then immersed in OCT medium (Sakura Finetek), frozen using dry ice, and sectioned at 14$\mu \mathrm{m}$ thickness. Nuclei were stained with Hoechst, and fluorescence microscopy of sections was performed on an EVOS FL Auto 2 Imaging System to evaluate zsG and tdT expression.

To analyze cell distribution of metastases from each primary tumor, individual micrometastases were identified in the lung. Micrometastases were identified as clusters of cells with a diameter larger than $200 \mu \mathrm{m}$, distinct from isolated tumor cells with a diameter less than $200 \mu \mathrm{m}$. The number of micrometastases composed of cells expressing zsG only, tdT only, or both zsG and tdT were manually counted in 5 fields of view per lung section for 3 lung sections for two mice sacrificed on day 42 and four mice sacrificed on day 38 .

\section{Statistics}

Linear regression analysis was performed to determine the goodness-of-fit coefficient ( $R^{2}$ value) for BLI signal versus number of Akaluc- or Antares2-expressing cells. Unpaired two-tailed $t$ tests were performed using GraphPad Prism software (Version 8.1.2 for Mac OS X, GraphPad Software Inc., La Jolla California, USA, www.graphpad.com) to compare BLI signal from Akaluc- and Antares2-expressing cells for in vitro and in vivo experiments. A $p$-value less than 0.05 was considered statistically significant. For cell proliferation and imaging measurements over time, one-way ANOVA and Tukey's multiple comparison post hoc test was performed.

\section{Results}

\section{Antares2 and Akaluc Do Not Exhibit Substrate Cross-Reactivity}

We first generated lentiviral vectors encoding either a pEF1 $\alpha$-zsG/Antares 2 or a pEF1 $\alpha$-tdT/Akaluc cassette (Fig. 1a). Human breast cancer cells were transduced with each lentivirus and sorted for $\mathrm{ZsG}$ or tdT to obtain Antares2- and Akaluc-expressing cell populations with $>95 \%$ purity (Fig. 1b). Fluorescence microscopy also confirmed zsG and tdT expression in purified Antares2-expressing and Akalucexpressing cell populations, respectively (Fig. 1c). As expected, Antares2-expressing cells also exhibited some red fluorescence due to CyOFP1 in the fusion protein. To test for Antares 2 and Akaluc functionality, increasing numbers of cells were seeded in a well plate, and BLI signal was measured after co-incubation with the appropriate substrate (Fig. 1d/e). A significant linear correlation was found between BLI signal and the number of both Antares2expressing cells (Fig. 1d; $n=3, R^{2}=0.9810, p<0.0001$ ) and Akaluc-expressing cells (Fig. 1e; $n=3, R^{2}=0.9944$, $p<0.0001)$. Cell proliferation assay of naïve cells, Antares2-expressing cells, and Akaluc-expressing cells over a 96-h period showed that there were no significant differences in cell growth at any of the time points (Fig. 1f).

To validate the use of Antares2 and Akaluc reporters for dual-BLI of SBBC growth and metastasis, we first needed to confirm a lack of substrate cross-reactivity of furimazine with Akaluc and AkaLumine-HCl with Antares2. Akalucexpressing cells, Antares2-expressing cells, naïve cells, and an equivalent volume of media were incubated with furimazine. Antares2-expressing cells showed significantly higher BLI signal $\left(10^{3}\right.$-fold $)$ than Akaluc-expressing cells, naïve cells, and media (Supplementary Fig. 1A; $n=3$, $p<0.05)$. Similarly, when treated with AkaLumine-HCl, Akaluc-expressing cells showed a significantly higher BLI signal $\left(10^{3}\right.$-fold) than Antares2-expressing cells, naïve cells, and media (Supplementary Fig. 1B; $n=3, p<0.01$ ). We next characterized the in vitro signal kinetics to determine the time for Antares2 and Akaluc signal to decay to background levels. Antares2- and Akaluc-expressing cells were treated with the appropriate substrate and imaged 5 min later. Cells were then washed with PBS and incubated in fresh media without substrate, and images were acquired over time with additional washes every $24 \mathrm{~h}$ until negligible signal remained. Antares2-expressing cells showed negligible signal remaining by $24 \mathrm{~h}$ after substrate treatment (Supplementary Fig. 1C). In contrast, Akaluc-expressing cells showed some signal remaining at $24 \mathrm{~h}$ but negligible signal by $48 \mathrm{~h}$ (Supplementary Fig. 1D). This data suggests that Antares 2 and Akaluc dual-BLI can be performed on consecutive days for in vitro experiments, with Akaluc BLI following Antares2 BLI.

We next evaluated the substrate cross-reactivity between Antares2 and Akaluc in vivo. Nude mice were implanted with either Antares2- or Akaluc-expressing cells into the mammary fat pad ( $n=4$ per cell line). We allowed the tumors to grow and become palpable before conducting crossreactivity tests. On day 5 , furimazine was injected intravenously in all eight mice, and BLI signal from mammary fat pad tumors were measured. On day 6, prescans confirmed the loss of Antares2 signal if previously present, and AkaLumine- $\mathrm{HCl}$ was injected intraperitoneally in all mice, and BLI signal was measured. Antares2 tumors showed significantly higher signal $\left(10^{2}\right.$-fold $)$ than Akaluc tumors when mice were injected with furimazine (Supplementary Fig. 2A; $n=4, p<0.01)$. Similarly, Akaluc-expressing tumors showed significantly higher signal $\left(10^{4}\right.$-fold) than Antares2expressing tumors after injection of AkaLumine- $\mathrm{HCl}$ (Supplementary Fig. 2B; $n=4, p<0.01$ ). These data validate the lack of substrate cross-reactivity between Antares 2 and Akaluc both in vivo and support their use in dual-BLI. For in vivo kinetics studies, nude mice bearing Antares2 or Akaluc mammary fat pad tumors were injected with the appropriate substrate and imaged over time until negligible signal remained. Antares2 BLI signal peaked immediately and dropped to background levels by $3 \mathrm{~h}$ after furimazine 
a

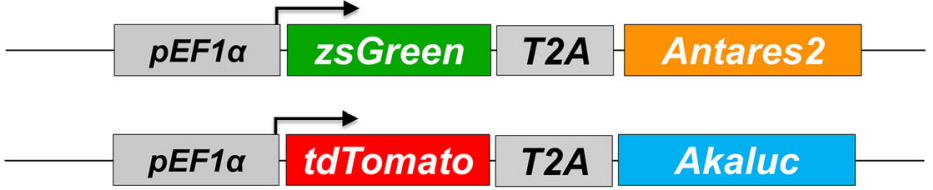

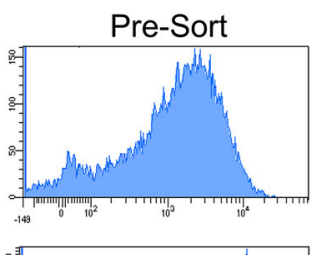

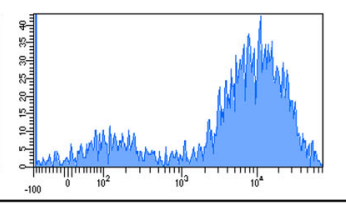

Fluorescence Intensity
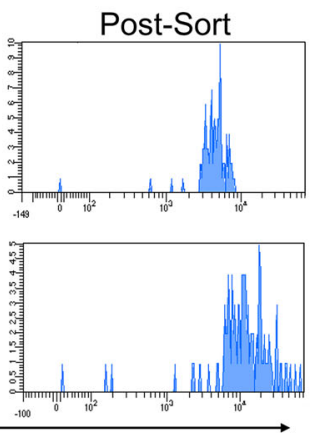

C

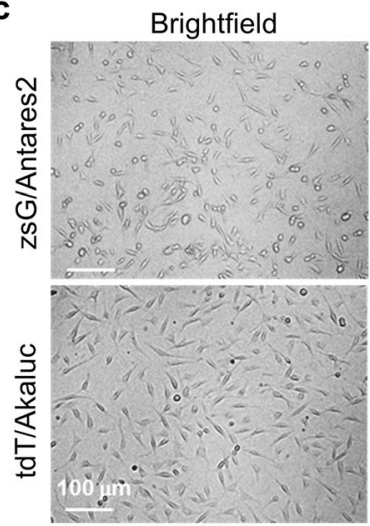

$594 / 630$

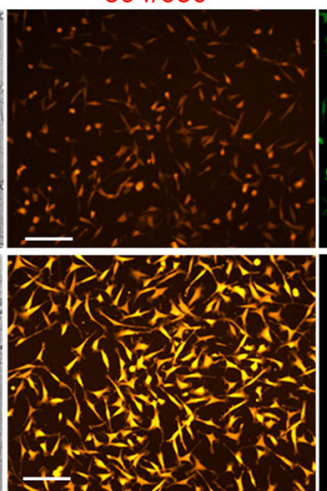

$488 / 520$
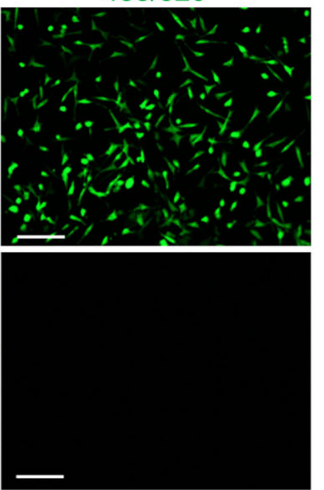

d
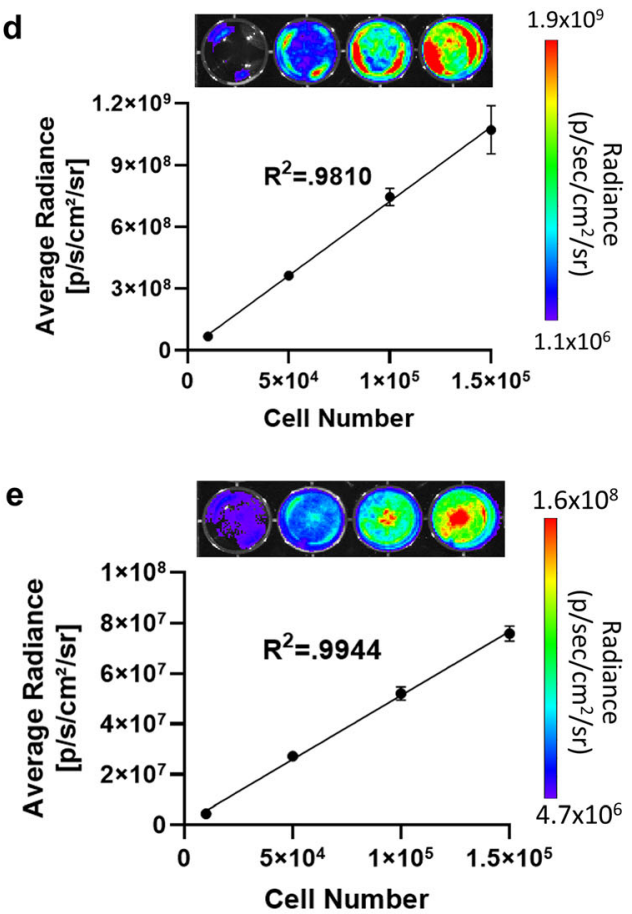

f

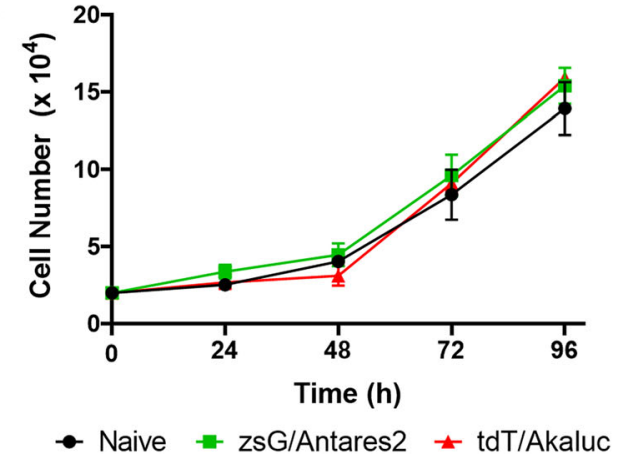

Figure. 1. Lentiviral transduction of MDA-MB-231 cells to express fluorescent and bioluminescent reporter genes: a Reporter gene constructs for co-expression of zsGreen (zsG) and Antares2, and tdTomato (tdT) and Akaluc. b Histograms of control (naïve) and transduced MDA-MB-231 cells fluorescence-activated cell sorted for ZsG or for tdT. c Fluorescence microscopy of sorted MDA-MB-231 cells engineered to express zsG/Antares2 or tdT/Akaluc, with excitation laser/emission filter wavelengths. d Bioluminescence imaging (BLI) signal vs number of Antares2-expressing cells after administration of furimazine. e BLI signal vs number of Akaluc-expressing cells after administration of AkaLumine- $\mathrm{HCl}$. $\mathbf{f}$ Cellular proliferation of naïve cells, Antares2expressing cells, and Akaluc-expressing cells. The data are presented as mean \pm SD.

injection (Supplementary Fig. 2C). In contrast, Akaluc BLI signal peaked at $30 \mathrm{~min}$ and dropped to background levels by $36 \mathrm{~h}$ after AkaLumine- $\mathrm{HCl}$ injection (Supplementary Fig. 2D). From these results, in vivo Antares2 and Akaluc dualBLI could be performed on the same day or on consecutive days, with Akaluc BLI following Antares2 BLI.

\section{Dual-BLI of Contralateral Tumors Shows Spontaneous Metastasis of Both Antares2- and Akaluc-Expressing Cells to the Lungs}

To generate a SBBC mouse model, Antares2-expressing cells were implanted into the right mammary fat pad and
Akaluc-expressing cells into the left mammary fat pad of ten NSG mice. NSG mice were chosen for our SBBC model as they allow for a more accurate representation of spontaneous metastasis seen in human breast cancer patients in comparison to nude mice $[21,22]$. We had six mice in our first cohort (mice 1-6) and four mice in a second cohort (mice 710). Dual-BLI was performed on the same or consecutive days weekly to track the growth of primary tumors and development of spontaneous metastases. Mice were imaged until endpoint as determined by the first presence of both Antares2 and Akaluc BLI signal in the lungs (day 29 for mice 1-3) or when evidence of ulceration of the primary tumors was present (day 38 for mice 7-10 and day 42 for mice 4-6). 
a

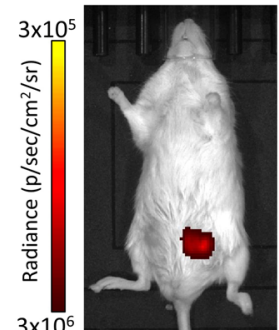

Day 0 Antares2

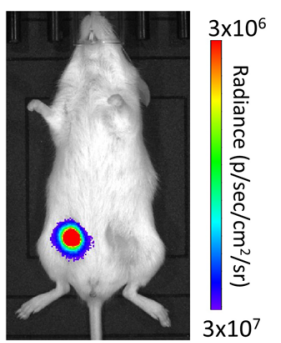

Day 1 Akaluc b

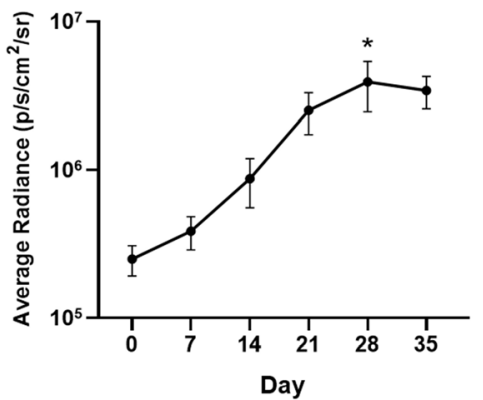

d

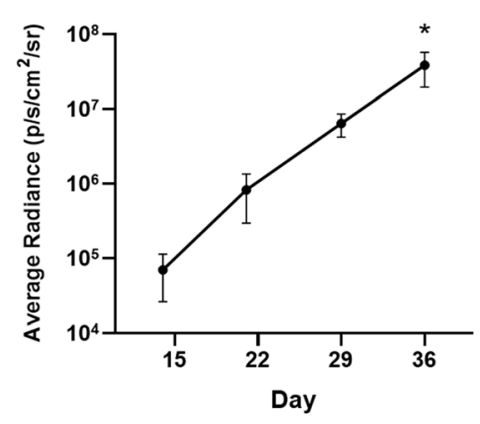

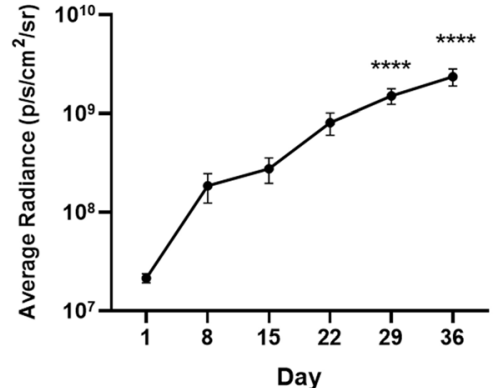

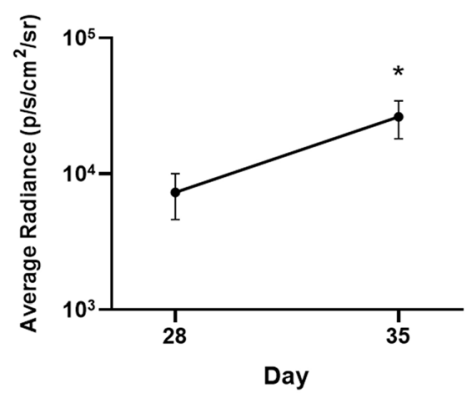

Figure. 2. Dual-bioluminescence imaging (BLI) of mammary fat pad tumors and lung metastasis in NOD scid-gamma mice: a Representative images of a mouse bearing contralateral mammary fat pad tumors imaged with Antares2 BLI on day 0 and Akaluc BLI on day 1. b Quantification of Antares2 (left) and Akaluc (right) mammary fat pad tumor BLI signal over time $(n=10)$. c Representative images of Akaluc and Antares2 BLI of lung metastasis. d Quantification of Akaluc (left) and Antares2 (right) lung BLI over time $(n=10) .{ }^{*} p<0.05,{ }^{* \star \star \star} p<0.0001$ when compared to the initial data point. The data are presented as mean \pm SEM.

On day 0 , only the Antares 2 tumor was visualized upon administration of furimazine, and on day 1, only the Akaluc tumor was visualized upon administration of AkaLumine$\mathrm{HCl}$ (Fig. 2a). Average radiance of BLI signal significantly increased over time for both Antares 2 and Akaluc mammary fat pad tumors (Fig. $2 \mathrm{~b} ; p<0.05$ ).

Metastases were first detected in the lungs in six of the 10 mice on day 15 using Akaluc BLI, with all mice showing Akaluc signal in the lungs by day 29 (Fig. 2c). Antares2-BLI detected lung metastasis in four of the 10 mice by day 28 and in six of the seven mice on day 35. By endpoint, nine of the 10 mice showed both Antares2 and Akaluc signal in the lungs. Average radiance significantly increased over time for both Antares 2 and Akaluc BLI signal from the lungs (Fig. $2 \mathrm{~d}, p<0.05)$.

\section{A High Percentage of Micrometastases Are Composed of Cancer Cells from Both Primary Tumors}

At endpoint, primary tumors and lungs were collected for analysis. Of the 10 mice, one mouse (mouse 4) was not preserved properly and thus excluded from further analysis. Akaluc primary tumors showed only tdT fluorescence, while Antares 2 primary tumors showed only zsG fluorescence (Supplementary Fig. 3). We believe the absence of visible orange-red CyOFP1 fluorescence in Antares2-expressing cells is due to the perfusion fixation of the mice.

The lungs of the 3 mice sacrificed on day 29 showed both zsG- and tdT-expressing cancer cells, which were mostly found in unique loci and isolated from each other (Fig. 3a/b). No micrometastases, defined as lesions with a diameter larger than $200 \mu \mathrm{m}$, had yet developed in these lungs, yet both Antares 2 and Akaluc signal was clearly visible. For mice sacrificed on day 38 (mice 7 through 10), there were micrometastases present, with many composed of zsGexpressing cells, a moderate fraction composed of both zsG- and tdT-expressing cells, and a small fraction composed solely of tdT-expressing cells (Fig. 3a/b, Supplementary Fig. 4). Finally, for the two mice sacrificed on day 42 (mice 5 and 6), many micrometastases were present in the lungs, with a large fraction qualitatively composed of both zsG- and tdT-expressing cells (Fig. 3a/b).

To quantify the composition of micrometastases, the number of lung micrometastases in mice 5 through 10 composed of only tdT-expressing cells, only zsG-expressing cells, or both cell types was manually counted in five fields of view in each of three lung sections (Fig. 4a). For mice 710 sacrificed on day 38 , the mean percentage of micrometastases composed of both cell types was $37 \%$, with percentages ranging from 27 to $45 \%$ (Fig. 4b). For mice 5 and 6 sacrificed on day $42,51 \%$ and $70 \%$ of micrometastases were composed of both cell types, respectively. 
a

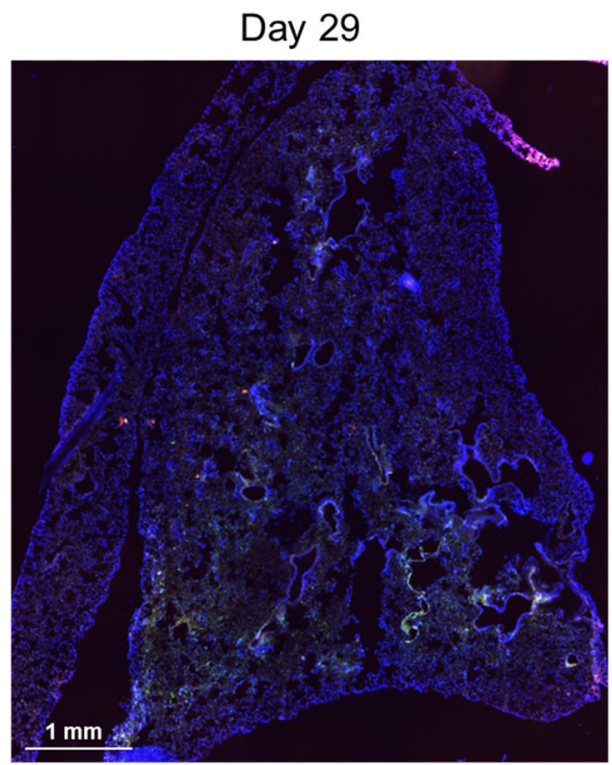

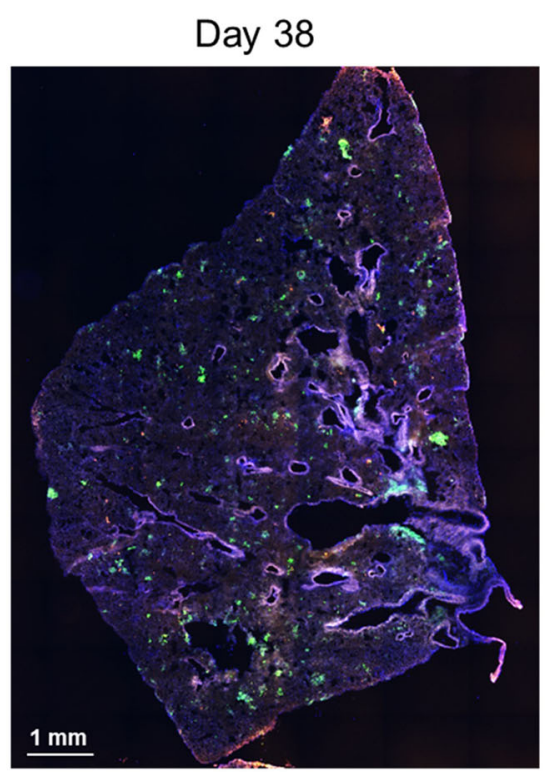

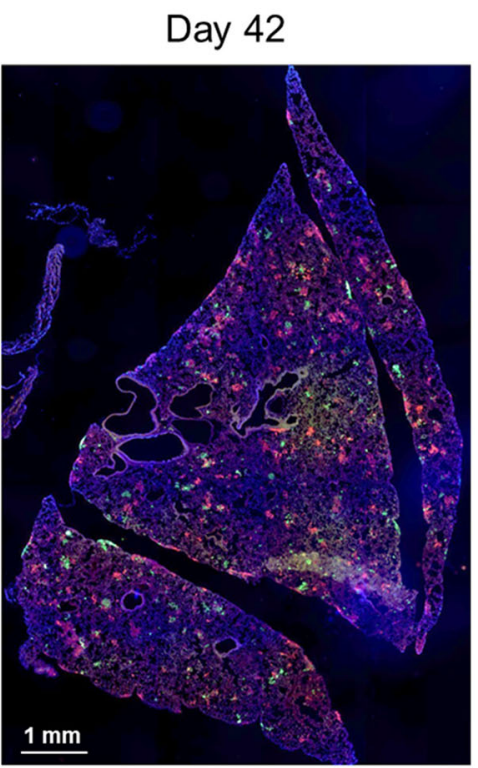

b

Hoechst

tdTomato

zsGreen

Merge

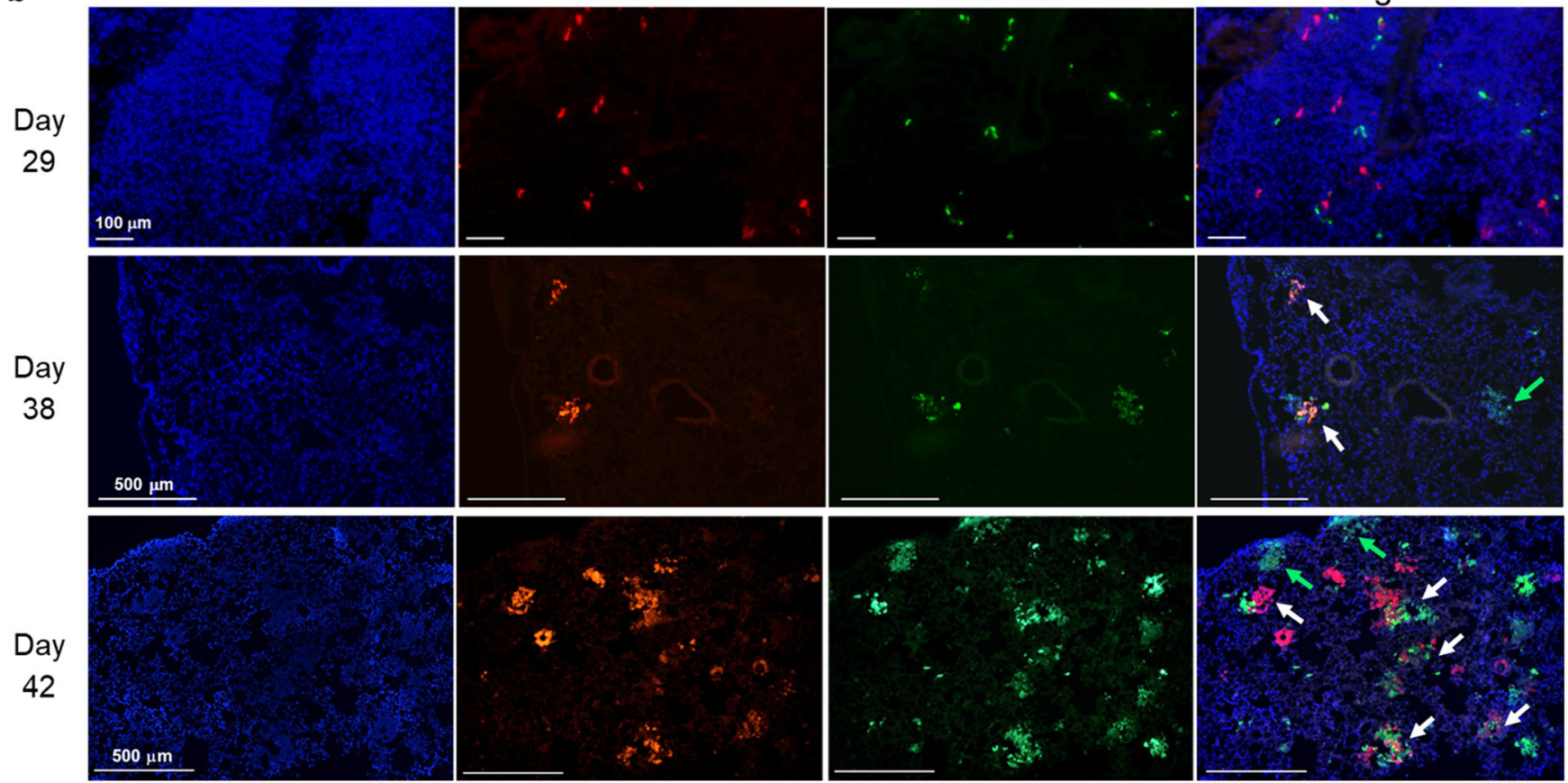

Figure. 3. Fluorescence microscopy lungs of mice sacrificed on day 29, day 38 , and day 42: a Representative whole lung sections of mice sacrificed at the three endpoints showing the presence of metastatic tdTomato (tdT) and zsGreen (zsG)expressing cells. b Higher magnification images (10x) showing micrometastases composed of cells derived from both mammary fat pad tumors (white arrows) and micrometastases composed of only zsG-expressing cells (green arrows). No micrometastases composed of only tdT-expressing cells were identified in these fields of view.

When averaged across all six mice, there was a significantly higher percentage of micrometastases composed of both cell types compared to the percentage of micrometastases composed of only tdT-expressing cells (Fig. 4c; $p<0.001$ ). We noticed that for mice $7-10$, there was a large proportion of zsG-only micrometastases compared to tdT-only micrometastases. We then analyzed the primary tumor masses of the mice at endpoint and found that while mice 5 and 6 had similar zsG/Antares2 and tdT/Akaluc tumor masses, for mice 7-10, the masses of the $\mathrm{zsG} /$ Antares 2 tumors were significantly higher than the masses of the tdT/ Akaluc tumors (Fig. 4d/e; $p<0.001$ ), likely explaining the 

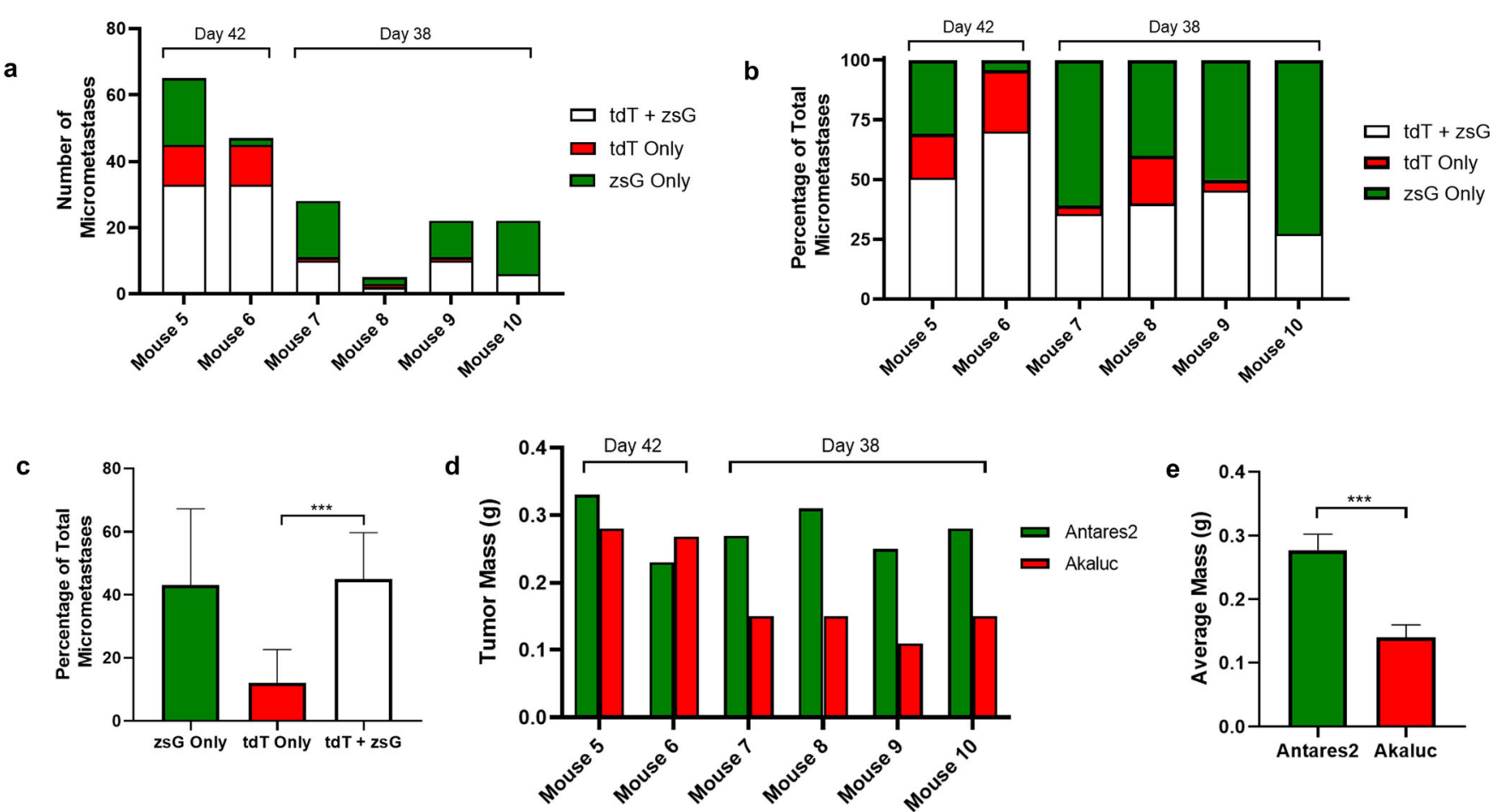

Figure. 4. Cell composition analysis of lung micrometastases. The number of micrometastases $(>200 \mu \mathrm{m}$ diameter) composed of only zsGreen (zsG)-expressing cells, only tdTomato (tdT)-expressing cells, or both were counted in 5 fields of view at 3 lung sections for mice 5-6 (sacrificed on day 42) and mice 7-10 (sacrificed on day 38). Quantification of the types of micrometastases is expressed as total counts (a) or percentages of the total count (b). (c) Mean percentages of micrometastases types for mice $5-10\left(n=6,{ }^{\star \star *} p<0.001\right)$. (d) Mammary fat pad tumor masses for individual mice at endpoint. (e) Average mammary fat pad tumor masses for mice $7-10\left(n=4,{ }^{* \star} p<0.001\right)$. The data are presented as mean \pm SD.

high percentage of zsG-only micrometastases in this particular cohort.

\section{Discussion}

SBBC is being presented to clinicians as an emerging concern, with increasing incidence due to improved screening technologies, prolonged life expectancy, and a growing awareness of the disease $[1,3]$. Several retrospective clinical studies indicate that SBBC patients have higher rates of distant metastasis and higher mortality rates [2,3]. However, due to the lack of longitudinal data characterizing the growth of SBBC metastases, little is known about the patterns of metastasis, undermining our ability to understand why SBBC patients are at increased risk of death. Characterizing the dynamics of SBBC metastasis will deepen our understanding of this disease and may eventually offer insights into mechanisms that may be targeted by anti-metastatic therapies, treatment resistance, and ways to better manage SBBC patients.

In this study, we developed the first animal model to characterize metastatic SBBC. We genetically engineered human breast cancer cells to express either Antares 2 or Akaluc for BLI and zsG or tdT for fluorescence microscopy. We then implanted these cell populations bilaterally into contralateral mammary fat pads of mice and used in vivo
dual-BLI and ex vivo fluorescence microscopy to analyze the cell distribution of lung metastases arising from each mammary fat pad tumor. Akaluc BLI first detected lung metastasis on day 15, whereas Antares2 BLI first detected lung metastasis on day 28. This is believed to be due to the lower sensitivity of the Antares2 reporter in vivo. By their respective endpoints, nine of 10 mice showed metastasis of both mammary fat pad tumors to the lungs. Analysis of the locations of these cells in mice sacrificed on day 42 showed that a majority of micrometastases in the lungs were composed of both zsG- and tdT-expressing cells, indicating metastasis of cells from both primary tumors to the same locations or the cross-seeding between zsG or tdT lung metastases once formed. We then performed this experiment again due to having only two mice sacrificed on day 42 . In this second cohort of mice, due to the size of the primary tumors, these mice were sacrificed on day 38. Again, at endpoint we saw a significant portion of micrometastases composed of both zsG- and tdT-expressing cells, but compared to mice sacrificed on day 42 , more micrometastases were composed of only zsG-expressing cells. We believe this to be due to faster-growing zsG/Antares2 mammary fat pad tumors in this particular cohort compared to the first cohort, as shown by a significantly higher average primary tumor mass at endpoint. When averaged across all six mice, we found that the percentage of micrometastases 
composed of both cell types was significantly higher than the percentage of micrometastases composed of only tdTexpressing cells.

Our results provide new information on the patterns of metastasis in an SBBC mouse model, suggesting that two contralateral tumors may preferentially co-populate individual metastases in a process we call metastatic cross-seeding. This is similar to a phenomenon known as tumor selfseeding, in which circulating tumor cells (CTCs) that disseminate from a primary tumor travel through the circulation and seed back to the original tumor $[23,24]$. This process has been hypothesized to be due to leaky microvasculature of the original tumor, providing a favorable environment to infiltrate into and populate. Similarly, CTCs have been shown to seed onto established metastatic sites possessing favorable survival conditions [25-27]. We have recently found that systemically administered CTCs can readily self-home to pre-established spontaneous metastases that have disseminated throughout the body and used this to develop a novel cell-based theranostic [27]. Another possibility is that cells from metastases in the lungs disseminate again and seed onto other metastases in the organ, further contributing to mixed zsG- and tdT expression within the same tumor. In the future, it will be of value to study this phenomenon with more invasive imaging technologies, such as intravital fluorescence microscopy, in order to visualize this process in real time. These mechanisms supporting the metastatic cross-seeding phenomenon may be an explanation for the current study's findings that cells are more likely to seed onto pre-established metastases, contributing to a high percentage of lung metastases composed of cells that originated from distinct primary tumors.

The cross-seeding of contralateral tumors may help explain the accelerated growth of SBBC metastasis and ultimately the poorer outcomes shown by retrospective clinical studies. Importantly, if the primary cancers are discordant in tumor biology including histopathologic classification, mutation status, hormone receptor status, and HER2 receptor status, metastatic cross-seeding may lead to increased intratumoral heterogeneity, adding another layer of complexity when choosing the optimal treatment plan. A study of the pathological profiles of 2542 SBBC patients found estrogen receptor status, progesterone receptor status, and HER2 status discordance rates between the two primary tumors of $14.2 \%, 23.4 \%$, and $14.6 \%$, respectively, with an overall molecular subtype discordance rate of $24.2 \%$ [28]. With these factors being established biomarkers for expected treatment response and prognosis, hormone receptor discordance in SBBC patients has been associated with increased rate of metastasis and higher mortality at 5 years $[29,30]$. A cohort study on the effect of adherence to the German national S3-guideline for adjuvant therapy on the survival outcome of bilateral breast cancer patients showed that patients who were treated guideline-adherent for only the primary index tumor suffered a significantly decreased recurrence-free survival compared to patients treated guideline-adherent for both tumors [31]. Although this study shows a need to maximize targeting of both lesions, the risk of adverse side effects of rigorous therapy plans must also be considered. The limited scientific evidence on the disease and a current lack of international guidelines on therapeutic management calls for a need to further investigate the properties of SBBC and the impact of various treatment plans on survival.

A limitation of our work is that we only characterized the cellular composition of metastases in the lungs where cells were located using in vivo BLI. Besides the lungs, SBBC patients also commonly show metastasis to the bone, liver, brain, and axillary lymph nodes $[2,5]$. In our model, the growth of the primary tumors hindered our ability to image for a longer period of time. Surgically removing the primary tumors may allow us to prolong our study until metastasis in other organs can be detected. This has previously been demonstrated in a mouse model of breast cancer, where removal of the primary tumor extended the life span of mice but did not inhibit metastasis to distant organs [32]. Enhancing the sensitivity of our BLI reporters may also enable detection of other metastases. The sensitivity of Antares 2 could be improved by pairing it with diphenylterazine (DTZ), a more optimal substrate [15]. Akaluc BLI could be improved by increasing the concentration of its substrate AkaLumine- $\mathrm{HCl}$. By improving the sensitivity of Antares 2 and Akaluc and covering the primary tumors and lungs to avoid saturation of the CCD camera, it may be possible to detect metastasis in other organs. Another limitation is that Antares 2 shows some red fluorescence due to the presence of the orange fluorescent protein CyOFP1 in the fusion protein, which is visible in in vitro images. However, fluorescence microscopy of the Antares2 tumor did not show any perceptible red fluorescence, which we believe is due to the perfusion fixation of the mice, leading to reduced CyOFP1 fluorescence intensity. In addition, tdT-expressing cells do not show any green fluorescence, so all red fluorescence from lung sections is from tdT.

Future preclinical research on SBBC will aim to study the generalizability of the cross-seeding mechanism to other cell lines and to explore the effect of primary tumor discordance in hormone receptor and HER2 status. Our model consisted of concordant triple-negative breast cancer primary tumors, while in the clinic, it has been shown that only $3.3-3.6 \%$ of SBBC patients have this phenotype [28]. A large proportion of SBBC patients have discordant tumor molecular subtypes (e.g., HER2+/HER2-), and it is therefore of great interest to develop more clinically relevant models. Models consisting of cells taken directly from both primary tumors in SBBC patients may be more biologically relevant, although patientderived xenograft (PDX) models are difficult to establish and the ability to establish PDX in both mammary fat pads may be even more difficult [33]. Future work in clinical research of SBBC could involve genome sequencing of 
tumors to track the lineages of metastatic cells. Mutational profiling has previously been performed to determine whether primary tumors in SBBC patients were of independent or clonal origin, which may have clinical implications [34]. Similarly, gene sequencing techniques could also be applied to elicit the origin of metastatic tumors and determine their heterogeneity.

\section{Conclusions}

In this work, we established the first animal model of SBBC and evaluated the metastatic cell distribution of contralateral mammary fat pad tumors using in vivo dual-BLI and ex vivo fluorescence microscopy. We found that the majority micrometastases in the lungs are composed of cells from both primary tumors, suggesting a high degree of metastatic cross-seeding which we posit may contribute to intratumoral heterogeneity and treatment resistance. Our work deepens our understanding of the mechanisms underlying poor outcomes of SBBC patients and may offer insight into optimal management of SBBC.

Supplementary Information. The online version contains supplementary material available at https://doi.org/10.1007/s11307-021-01630-z.

Author Contribution. SL, NNN, and JAR contributed to the study conception and design. SL, NNN, JJK, and AMH carried out the experimental work. YF performed data analysis and validation. SL wrote the original draft, and all other authors reviewed and edited the manuscript. All authors have read and agreed to the published version of the manuscript.

Funding. This research was funded by a Canadian Institutes of Health Research (CIHR) Project Grant held by J.A.R. (\#377071). S.L. was supported by an Undergraduate Student Research Award from the Natural Sciences and Engineering Research Council of Canada.

\section{Declarations}

\section{Conflict of Interest}

The authors declare that they have no conflict of interest.

Open Access This article is licensed under a Creative Commons Attribution 4.0 International License, which permits use, sharing, adaptation, distribution and reproduction in any medium or format, as long as you give appropriate credit to the original author(s) and the source, provide a link to the Creative Commons licence, and indicate if changes were made. The images or other third party material in this article are included in the article's Creative Commons licence, unless indicated otherwise in a credit line to the material. If material is not included in the article's Creative Commons licence and your intended use is not permitted by statutory regulation or exceeds the permitted use, you will need to obtain permission directly from the copyright holder. To view a copy of this licence, visit http:// creativecommons.org/licenses/by/4.0/.

\section{References}

1. Pan B, Xu Y, Zhou YD, Yao R, Wu HW, Zhu QL, Wang CJ, Mao F, Lin Y, Shen SJ, Sun Q (2019) The prognostic comparison among unilateral, bilateral, synchronous bilateral, and metachronous bilateral breast cancer: a meta-analysis of studies from recent decade (20082018). Cancer Med 8(6):2908-2918

2. Jobsen JJ, van der Palen J, Ong F, Riemersma S, Struikmans H (2015) Bilateral breast cancer, synchronous and metachronous; differences and outcome. Breast Cancer Res Treat 153(2):277-283

3. Ozturk A, Alco G, Sarsenov D, Ilgun S, Ordu C, Koksal U, Nur Pilanci K, Erdogan Z, Izci F, Elbuken F, Agacayak F, Aktepe F, Ozmen V (2018) Synchronous and metachronous bilateral breast cancer: a long-term experience. J BUON 23(6):1591-1600

4. Beckmann KR, Buckingham J, Craft P, Dahlstrom JE, Zhang Y, Roder D, Stuart-Harris R (2011) Clinical characteristics and outcomes of bilateral breast cancer in an Australian cohort. Breast. 20(2):158164

5. Eliyatkin N, Zengel B, Yagci A, Comut E, Postaci H, Uslu A, Aktas S (2015) Properties of synchronous versus metachronous bilateral breast carcinoma with long time follow up. Asian Pac J Cancer Prev 16(12):4921-4926

6. Kheirelseid EA, Jumustafa H, Miller N, Curran C, Sweeney K, Malone $\mathrm{C}$ et al (2011) Bilateral breast cancer: analysis of incidence, outcome, survival and disease characteristics. Breast Cancer Res Treat 126(1):131-140

7. Mejdahl MK, Wohlfahrt J, Holm M, Balslev E, Knoop AS, Tjønneland A, Melbye M, Kroman N (2019) Breast cancer mortality in synchronous bilateral breast cancer patients. Br J Cancer 120(7):761767

8. Aslakson CJ, Miller FR (1992) Selective events in the metastatic process defined by analysis of the sequential dissemination of subpopulations of a mouse mammary tumor. Cancer Res 52(6):1399-1405

9. Liu L, Zhang SX, Liao W, Farhoodi HP, Wong CW, Chen CC et al (2017) Mechanoresponsive stem cells to target cancer metastases through biophysical cues. Sci Transl Med 9(400)

10. Lu J, Liu X, Liao YP, Wang X, Ahmed A, Jiang W, Ji Y, Meng H, Nel AE (2018) Breast cancer chemo-immunotherapy through liposomal delivery of an immunogenic cell death stimulus plus interference in the IDO-1 pathway. ACS Nano 12(11):11041-11061

11. Park MK, Lee CH, Lee H (2018) Mouse models of breast cancer in preclinical research. Lab Anim Res 34(4):160-165

12. Price JE, Polyzos A, Zhang RD, Daniels LM (1990) Tumorigenicity and metastasis of human breast carcinoma cell lines in nude mice. Cancer Res 50(3):717-721

13. Dothager RS, Flentie K, Moss B, Pan MH, Kesarwala A, PiwnicaWorms D (2009) Advances in bioluminescence imaging of live animal models. Curr Opin Biotechnol 20(1):45-53

14. Chu J, Oh Y, Sens A, Ataie N, Dana H, Macklin JJ, Laviv T, Welf ES, Dean KM, Zhang F, Kim BB, Tang CT, Hu M, Baird MA, Davidson MW, Kay MA, Fiolka R, Yasuda R, Kim DS, Ng HL, Lin MZ (2016) A bright cyan-excitable orange fluorescent protein facilitates dual-emission microscopy and enhances bioluminescence imaging in vivo. Nat Biotechnol 34(7):760-767

15. Yeh HW, Karmach O, Ji A, Carter D, Martins-Green MM, Ai HW (2017) Red-shifted luciferase-luciferin pairs for enhanced bioluminescence imaging. Nat Methods 14(10):971-974

16. Iwano S, Sugiyama M, Hama H, Watakabe A, Hasegawa N, Kuchimaru T, Tanaka KZ, Takahashi M, Ishida Y, Hata J, Shimozono S, Namiki K, Fukano T, Kiyama M, Okano H, Kizaka-Kondoh S, McHugh TJ, Yamamori T, Hioki H, Maki S, Miyawaki A (2018) Single-cell bioluminescence imaging of deep tissue in freely moving animals. Science. 359(6378):935-939

17. Kang JH, Chung JK (2008) Molecular-genetic imaging based on reporter gene expression. J Nucl Med 49(Suppl 2):164S-179S

18. Dull T, Zufferey R, Kelly M, Mandel RJ, Nguyen M, Trono D, Naldini L (1998) A third-generation lentivirus vector with a conditional packaging system. J Virol 72(11):8463-8471

19. Nyström NN, Hamilton AM, Xia W, Liu S, Scholl TJ, Ronald JA (2019) Longitudinal visualization of viable cancer cell intratumoral distribution in mouse models using Oatpla1-enhanced magnetic resonance imaging. Investig Radiol 54(5):302-311

20. Stacer AC, Nyati S, Moudgil P, Iyengar R, Luker KE, Rehemtulla A, Luker GD (2013) NanoLuc reporter for dual luciferase imaging in living animals. Mol Imaging 12(7):1-13

21. Puchalapalli M, Zeng X, Mu L, Anderson A, Hix Glickman L, Zhang M, Sayyad MR, Mosticone Wangensteen S, Clevenger CV, Koblinski JE (2016) NSG mice provide a better spontaneous model of breast 
cancer metastasis than athymic (nude) mice. PLoS One 11(9):e0163521

22. Iorns E, Drews-Elger K, Ward TM, Dean S, Clarke J, Berry D, Ashry DE, Lippman M (2012) A new mouse model for the study of human breast cancer metastasis. PLoS One 7(10):e47995

23. Kim MY, Oskarsson T, Acharyya S, Nguyen DX, Zhang XH, Norton L et al (2009) Tumor self-seeding by circulating cancer cells. Cell. 139(7):1315-1326

24. Wang WC, Zhang XF, Peng J, Li XF, Wang AL, Bie YQ et al (2018) Survival mechanisms and influence factors of circulating tumor cells. Biomed Res Int 2018:6304701

25. Dondossola E, Dobroff AS, Marchiò S, Cardó-Vila M, Hosoya H, Libutti SK, Corti A, Sidman RL, Arap W, Pasqualini R (2016) Selftargeting of TNF-releasing cancer cells in preclinical models of primary and metastatic tumors. Proc Natl Acad Sci U S A 113(8):2223-2228

26. Reinshagen C, Bhere D, Choi SH, Hutten S, Nesterenko I, Wakimoto $\mathrm{H}$ et al (2018) CRISPR-enhanced engineering of therapy-sensitive cancer cells for self-targeting of primary and metastatic tumors. Sci Transl Med 10(449)

27. Parkins KM, Dubois VP, Kelly JJ, Chen Y, Knier NN, Foster PJ, Ronald JA (2020) Engineering circulating tumor cells as novel cancer theranostics. Theranostics. 10(17):7925-7937

28. Ding S, Sun X, Lu S, Wang Z, Chen X, Shen K (2021) Association of molecular subtype concordance and survival outcome in synchronous and metachronous bilateral breast cancer. Breast. 57:71-79
29. Baretta Z, Olopade OI, Huo D (2015) Heterogeneity in hormonereceptor status and survival outcomes among women with synchronous and metachronous bilateral breast cancers. Breast. 24(2):131-136

30. Dhadlie S, Whitfield J, Hendahewa R (2018) Synchronous bilateral breast cancer: a case report of heterogeneous estrogen receptor status. Int J Surg Case Rep 53:102-106

31. Schwentner L, Wolters R, Wischnewsky M, Kreienberg R, Wöckel A (2012) Survival of patients with bilateral versus unilateral breast cancer and impact of guideline adherent adjuvant treatment: a multicentre cohort study of 5292 patients. Breast. 21(2):171-177

32. Paschall AV, Liu K (2016) An orthotopic mouse model of spontaneous breast cancer metastasis. J Vis Exp 114:54040

33. Eyre R, Alférez DG, Spence K, Kamal M, Shaw FL, Simões BM, Santiago-Gómez A, Sarmiento-Castro A, Bramley M, Absar M, Saad Z, Chatterjee S, Kirwan C, Gandhi A, Armstrong AC, Wardley AM, O’Brien CS, Farnie G, Howell SJ, Clarke RB (2016) Patient-derived mammosphere and xenograft tumour initiation correlates with progression to metastasis. J Mammary Gland Biol Neoplasia 21(3-4):99109

34. Bao L, Messer K, Schwab R, Harismendy O, Pu M, Crain B, Yost S, Frazer KA, Rana B, Hasteh F, Wallace A, Parker BA (2015) Mutational profiling can establish clonal or independent origin in synchronous bilateral breast and other tumors. PLoS One 10(11):e0142487

Publisher's Note Springer Nature remains neutral with regard to jurisdictional claims in published maps and institutional affiliations. 